INNOVATIONS IN PRIMARY CARE

\title{
Radical Redesign: The Power of Team-Based Care
}

James Jerzak, MD

Ann Fam Med 2017;15:281. https://doi.org/10.1370/afm.2072.

\section{THE INNOVATION}

The electronic health record (EHR) brings tremendous advantages, but it also poses sizeable challenges for the medical profession. Its demands can overburden physicians, and increasingly complex patient care requires a new approach. Acknowledging this and preparing for the impending change to value-based care, Bellin Health has embarked on a systemwide transformation to advanced team-based care.

\section{WHO \& WHERE}

Following months of research and planning by administrators and clinicians, the care team at Bellin Health Ashwaubenon in Green Bay, Wisconsin launched a team-based care prototype in November 2014. Bellin Health Ashwaubenon is a hospitalowned family medicine practice of 14 clinicians (9 physicians, 1 physician assistant, and 4 advance practice nurse practitioners) and 70 staff members. Our radical redesign required the collaboration of core and extended care-team members, as well as significant administrative support.

\section{HOW}

Early on, we realized that a complete overhaul of our traditional patient care system was necessary. We first radically redesigned the office visit, empowering medical assistants and licensed practical nurses to take an enhanced role in appointments, providing in-room support, and completing most EHR work. Developing and implementing the training to empower these staff members was challenging but well worth the effort. It makes a tremendous difference for the clinician to be able to focus on the patient with no EHR distractions.

We also redesigned between-visit work with team management in mind. With the EHR, the default location for most tasks is the clinician's in-basket. A team approach allows employees

Conflicts of interest: author reports none.

\section{CORRESPONDING AUTHOR}

James Jerzak, MD

Bellin Health Ashwaubenon

1630 Commanche Ave.

Green Bay, WI 54313

james.jerzak@bellin.org to work at the top of their skill sets, helping us meet patients' needs more efficiently. Engineering this culture shift wasn't easy, but through training and support, we adopted a true team approach. (For more on the principles and concepts that guided our changes, see Supplemental Appendixes 1 and 2.)

To truly innovate, we needed to involve members of the extended care team in the care of complex patients. Case managers, clinical pharmacists, diabetes educators, registered nurse care coordinators, and behavioral health professionals provide intensive support for clinicians, enhancing care quality and patient engagement. Incorporating these individuals was challenging and required obtaining leadership buy-in for resources as well as developing new workflows to involve care team members in meaningful ways. To make these changes possible, we had to redesign our physical workspace to enable our teams to colocate, which greatly improved communication. Working sideby-side is more efficient-and more satisfying for the team.

\section{LEARNING}

The effects of this transition have been overwhelmingly positive. Patient satisfaction has never been higher, quality measures have improved, and patient engagement is greatly increased. We, the care team, have also reaped the benefits. Taking the burden of EHR work off the clinician has once again made practicing medicine enjoyable, and the empowerment the team feels in their new roles has made their jobs more rewarding as well.

Team-based care is not the goal, but rather our means to the end of effective population health management. This requires extensive infrastructure changes to foster a sustainable process that can be used throughout the health system. Our prototype began to expand in May 2015. Now, 42 clinicians in 8 locations use the team-based care model with the goal of spreading it to all of Bellin's 140 primary care clinicians in 32 locations by the end of 2018. The significant quality improvement and decrease in the cost of patient care gives us confidence we can successfully navigate the impending transition to value-based payments. We have also determined and are meeting the metrics needed to make this transition sustainable in a fee-for-service environment.

Key words: practice redesign; medical care team; value-based purchasing.

Author affiliation, an acknowledgment, and supplemental appendixes are available at http://www.AnnFamMed.orgl content/15/3/281/suppI/DC1. 